DOI: http://dx.doi.org/10.12957/demetra.2014.10544

\title{
Padronização de dietas enterais não industrializadas para uso domiciliar: a experiência de Campinas
}

\section{Standardization of non-industrialized enteral feeding for home care: the experience of Campinas city}

\author{
Marina Borelli \\ Márcia Josefa Lima de Sá Carneiro² \\ Daniela C. Arengui ${ }^{3}$ \\ Semíramis Martins Álvares Domene ${ }^{4}$ \\ ${ }^{1}$ Curso de Nutrição, Universidade Paulista. \\ Jundiaí-SP, Brasil. \\ ${ }^{2}$ Secretaria de Saúde do município de \\ Hortolândia-SP. Hortolândia-SP, Brasil. \\ ${ }^{3}$ Hospital Municipal Dr. Mário Gatti. \\ Campinas-SP, Brasil. \\ ${ }^{4}$ Curso de Nutrição, Departamento de Políticas \\ Públicas e Saúde Coletiva. Universidade Federal \\ de São Paulo. Santos-SP, Brasil. \\ Correspondência /Correspondence \\ Marina Borelli \\ E-mail:marinaborellip@gmail.com
}

\section{Resumo}

A terapia nutricional enteral domiciliar é uma realidade recente e cresce diante do aumento da expectativa de vida. Contempla pacientes do Sistema Único de Saúde, mas o fornecimento de suplementos nutricionais e dietas enterais não é garantido por esse sistema. $\mathrm{O}$ atendimento domiciliar tem a finalidade de prestar assistência humanizada e proporcionar melhor qualidade de vida aos indivíduos enfermos, promovendo educação em saúde junto aos familiares e cuidadores. Grupo formado por nutricionistas de hospitais públicos e privados com leitos do Sistema Único de Saúde e de Serviços de Atendimento Domiciliar do município de Campinas-SP se reuniu para a discussão da nutrição enteral prescrita pelos hospitais com a proposta de padronizar dietas enterais não industrializadas para alta hospitalar, visando à seleção de fórmulas que atendam às necessidades nutricionais, com menor custo em relação à industrializada, facilidade de preparo e favorecimento da orientação de alta e acompanhamento nutricional no domicílio. Foram padronizadas sete dietas, preparadas simulando a situação do domicílio e encaminhadas para análise bromatológica para determinação da composição centesimal. Os cálculos a partir de tabelas de composição de alimentos foram comparados aos resultados da análise bromatológica. A partir dos resultados obtidos, ajustes nas dietas serão realizados e este protocolo será divulgado nos hospitais do município e da região.

Palavras-chave: Terapia Nutricional. Nutrição Enteral. Alimentos Formulados. Atendimento Domiciliar. 


\section{Abstract}

Home enteral nutrition therapy is a recent phenomenon and is growing because of the increasing life expectancy. It is offered to patients in the Unified Health System, but the provision of nutritional supplements and enteral feeding is not guaranteed by this system. Home care aims to provide humane care and provides better quality of life for sick individuals by promoting health education with relatives and caregivers. Group of nutritionists of public and private hospitals in the Unified Health System and Home Care Services in the city of Campinas-SP, Brazil, discussed enteral nutrition prescribed in hospitals to standardize nonindustrialized enteral feeding for hospital discharge, aimed at the selection of formulas that meet nutritional needs with lower cost compared to industrialized, ease of preparation and orientation favoring high and home nutritional counseling. Seven diets were standardized, prepared simulating the household situation and sent for chemical analysis to determine their chemical composition. Calculations from food composition tables were compared to the results of chemical analysis. From the results obtained, adjustments in diet will be performed and this protocol will be released in hospitals of the city and region.

Key words: Nutrition Therapy. Enteral Nutrition. Formulated Food. Home Care Service.

\section{Introdução}

A terapia nutricional enteral domiciliar (TNED) é um tratamento considerado econômico e seguro, pois evita a contaminação hospitalar, diminui os gastos com internação e promove o convívio familiar e social do paciente., ${ }^{1,2}$

No Brasil, a TNED é uma realidade recente, principalmente para pacientes do Sistema Único de Saúde (SUS), mas o fornecimento de suplementos nutricionais e dietas enterais não é garantido pelo mesmo sistema. ${ }^{1}$ É importante ressaltar o fato de que a realidade domiciliar difere das condições hospitalares, sendo os fatores socioeconômicos, culturais e familiares determinantes para o estabelecimento da conduta dietética no domicílio. ${ }^{3,4}$

$\mathrm{O}$ atendimento domiciliar tem a finalidade de garantir assistência humanizada e proporcionar melhor qualidade de vida aos indivíduos enfermos, assim como promover educação em saúde junto aos familiares e cuidadores. ${ }^{5,6}$ 
O suporte nutricional é o conjunto de medidas necessárias para identificar e corrigir desvios nutricionais, a partir da identificação dos indivíduos portadores de distúrbio nutricional atual ou que estejam sob risco de desenvolvê-lo, e da orientação da terapia nutricional necessária e adequada a cada paciente, com a finalidade de minimizar as consequências negativas de desnutrição, obesidade e outras doenças associadas, garantindo a segurança alimentar pela nutrição enteral. ${ }^{6}$

A terapia nutricional enteral domiciliar é o tratamento acompanhado pelo nutricionista e indicado na prevenção da desnutrição e melhora do estado nutricional do paciente em uso de sonda enteral ou consumo via oral. É indicada para indivíduos cuja ingestão alimentar é inadequada, para prover suas necessidades nutricionais diárias, ou seja, que apresentam risco de desnutrição, sendo necessário que o trato digestório seja total ou parcialmente funcional. Estão incluídos também os indivíduos com patologias do TGI alto, distúrbios neurológicos com comprometimento do nível de consciência ou dos movimentos mastigatórios, pacientes intubados com anorexia de diversas etiologias e câncer. ${ }^{6,7}$

A TNED, quando prescrita corretamente, traz benefícios clínicos ao indivíduo, com redução do tempo de hospitalização e da incidência de complicações, bem como melhoria na qualidade de vida dos pacientes e na assistência prestada, gerando maior disponibilidade de leitos hospitalares e menores gastos com a saúde., ${ }^{2,6,7}$

De acordo com RDC/36 da Agência Nacional de Vigilância Sanitária, a nutrição enteral é definida como:

[...] alimento para fins especiais, com ingestão controlada de nutrientes, na forma isolada ou combinada, de composição definida ou estimada, especialmente formulada e elaborada para uso por sondas ou via oral, industrializado ou não, utilizada exclusiva ou parcialmente para substituir ou complementar a alimentação oral em pacientes desnutridos ou não, conforme suas necessidades nutricionais, em regime hospitalar, ambulatorial ou domiciliar, visando a síntese ou manutenção dos tecidos, órgãos ou sistemas. ${ }^{8}$

As dietas industrializadas são as mais indicadas por serem práticas, nutricionalmente completas e seguras quanto ao controle microbiológico. No entanto, devido ao alto custo, não são acessíveis para a maioria da população brasileira, sendo necessário indicar dietas não industrializadas com composição nutricional adequada, motivo pelo qual se buscam inovação e melhora da qualidade nutricional dessas formulações. Estima-se que, no Brasil, cerca de $50 \%$ dos hospitais trabalham com dietas industrializadas, embora as mesmas estejam disponíveis no mercado há mais de 20 anos. Também se discute o custo das dietas industrializadas. ${ }^{2,9,10}$ 
A formulação não industrializada é uma opção para fornecer macronutrientes para paciente em terapia nutricional enteral domiciliar com um tipo de alimentação que apresenta boa tolerância da maioria dos pacientes assistidos por equipe especializada. ${ }^{2}$ A oferta de micronutrientes, quando insuficiente para atingir as quantidades recomendadas, é parcialmente atendida via compostos mistos de minerais e vitaminas, sulfato ferroso e suplementação com sucos de frutas ou vitaminas e suplementos alimentares industrializados.

Com o aumento crescente da TNED, as formulações enterais não industrializadas fazem parte da orientação nutricional de pacientes de baixa renda, sendo elaboradas com alimentos e ingredientes convencionais, combinados ou não com fórmulas ou suplementos industrializados. As dietas não industrializadas também podem ser individualizadas de acordo com algumas necessidades ou demandas decorrentes de doenças que não são satisfeitas pela fórmula padrão, e exigem do nutricionista o exercício da técnica dietética em tentativas empíricas, devido ao escasso número de artigos e trabalhos científicos publicados na área. ${ }^{9}$ Quando possível, é mais adequado determinar a composição centesimal e de características físicas e físico-químicas $(\mathrm{pH}$, osmolalidade, viscosidade, estabilidade) das dietas em laboratórios especializados. Além disso, diversos trabalhos têm sido desenvolvidos na tentativa de definir formulações não industrializadas que possam ser empregadas com segurança nutricional e microbiológica na prática clínica. .,9,10 $^{2,10}$

De acordo com Mitne, ${ }^{11}$ dietas artesanais ou caseiras são definidas como aquelas "preparadas à base de alimentos in natura, produtos alimentícios e/ou módulos de nutrientes". A mesma autora refere que alimentos in natura são considerados os alimentos em seu estado natural, que têm como objetivo fornecer nutrientes, como leites, ovos, carnes, frango, legumes, verduras, leguminosas, cereais e frutas. Já os produtos alimentícios representam os alimentos que passaram por algum processo de industrialização, como suplementos proteicos, leites em pó, ovos na forma liofilizada, óleos vegetais, açúcar, amido de milho, dentre outros.

O módulo de nutrientes é caracterizado como produto alimentício que fornece um tipo de nutriente. Baxter \& Waitzberg ${ }^{12}$ complementam o conceito de dieta caseira quando relatam que esta é "liquidificada e preparada artesanalmente em cozinha doméstica ou hospitalar." Borges et al. ${ }^{13}$ corroboram tal ideia e ressaltam a necessidade de conscientização, educação e treinamento daqueles envolvidos com o preparo da dieta caseira em termos de terapia nutricional enteral domiciliar.

O Serviço de Atendimento Domiciliar (SAD) do município de Campinas tem como objetivo dar continuidade à prescrição dietética da alta hospitalar. Assim, surgiu a proposta de 
padronização de dietas enterais não industrializadas para alta hospitalar, visando à elaboração de fórmulas que atendam ao máximo as necessidades nutricionais, com menor custo em relação à industrializada, facilidade de preparo e favorecimento da orientação de alta e do acompanhamento nutricional no domicílio.

O objetivo deste artigo é apresentar os resultados de um processo de padronização de dietas não industrializadas realizado no município de Campinas.

\section{Métodos}

O processo de padronização das dietas iniciou-se no final de 2005, com uma reunião entre nutricionistas dos hospitais públicos e privados com leitos do SUS e dos serviços de atendimento domiciliar de Campinas-SP, para discussão da nutrição enteral prescrita pelos hospitais. O objetivo era padronizar as dietas enterais não industrializadas na alta hospitalar, em relação a custo e viabilidade, para facilitar a orientação nutricional no domicílio.

A partir dessa reunião, foi formado um grupo de trabalho de nutricionistas das seguintes unidades: SAD de Campinas, Hospital Municipal Mário Gatti (HMMG), Hospital e Maternidade Celso Pierro (HMCP/PUC-Campinas) e Hospital das Clínicas (HC/UNICAMP). A proposta foi aperfeiçoar as ações que envolvem a TNED, visando principalmente à elaboração de fórmulas que atendam às necessidades nutricionais, com menor custo em relação a dieta industrializada, facilidade de preparo e favorecimento da orientação de alta e do acompanhamento nutricional no domicílio.

O grupo de trabalho realizou reuniões mensais no ano de 2006 e discutiu as dietas a serem padronizadas a partir da prática de cada instituição e da prática do SAD, de acordo com as necessidades dos pacientes e das características das famílias (condição socioeconômica, cultural, nível de escolaridade). Os critérios para a determinação das sete dietas enterais não industrializadas foram: presença de alimentos com qualidade nutricional, praticidade, tempo de preparo, menor risco de contaminação e cálculo do valor econômico para identificar a dieta de menor custo.

A caracterização nutricional das formulações foi realizada pelo Laboratório de Micronutrientes da Faculdade de Nutrição / Centro Ciências da Vida, da Pontifícia Universidade Católica de Campinas (PUC-Campinas).

A escolha das dietas foi baseada nos seguintes critérios: qualidade, praticidade, tempo de preparo, menor risco de contaminação e menor custo. A partir dos critérios de escolha, as dietas foram definidas com base no seguinte esquema de orientação nutricional adotado pelo Serviço de Atendimento Domiciliar do município: 


\begin{tabular}{lll}
\hline \multicolumn{1}{c}{ Tipo de Dieta } & \multicolumn{1}{c}{ Características } & \multicolumn{1}{c}{ Indicações } \\
\hline GERAL & $\begin{array}{l}\text { Polimérica, Normocalórica } \\
\text { e Normoproteica }\end{array}$ & $\begin{array}{l}\text { Desnutrição } \\
\text { Pré e pós-operatórios }\end{array}$ \\
\hline Hipercalórica e & Hipercalórica & Necessidade elevada de \\
Hiperproteica & Hiperproteica & energia e restrição de \\
& & volume insuficiência \\
& & cardíaca \\
& Necessidade aumentada de \\
proteína
\end{tabular}

As dietas foram determinadas a partir dos cálculos nutricionais apresentados e critérios já citados. Para a realização deste cálculo, foram definidas as seguintes tabelas de composição de alimentos: TACO - 2006 ${ }^{14}$; USDA - 2006: ${ }^{15}$ e para fibras solúveis e insolúveis, a tabela Mendez et al., $1995^{16}$. A partir dos cálculos de composição centesimal, porcentagem de macronutrientes, densidade calórica e de custo das dietas, as dietas selecionadas foram preparadas em $1.000 \mathrm{ml}$ / 1.000 kcal de cada formulação e enviadas para a análise bromatológica. 
Para a análise de composição centesimal, as dietas foram preparadas de acordo com roteiro de padronização do preparo, utilizando sempre os mesmos equipamentos e utensílios em um único lactário, do Hospital e Maternidade Celso Pierro/PUC-Campinas, simulando o preparo da dieta no domicílio.

Após o preparo das dietas, as amostras congeladas foram então liofilizadas (Flexy-Dry Freeze Dryer, Kinetics, USA), sendo submetidas em análise duplicata nas seguintes determinações de composição centesimal:

- umidade, por gravimetria, conforme método proposto por Pearson; ${ }^{17}$

- proteína bruta, a partir da determinação de nitrogênio pelo método semimicro Kjeldahl ${ }^{18}$, empregando-se fatores de conversão de nitrogênio específicos $;^{19}$

- lípides totais, por extração dupla em metanol e clorofórmio, conforme procedimento proposto por Bligh \& Dyer; ${ }^{20}$

- cinzas, por calcinação seguida de incineração em mufla; ${ }^{21}$

- carboidratos, por diferença.

\section{Resultados}

As dietas padronizadas têm como base principal o extrato de soja e ingredientes de baixo custo, para que sejam viáveis economicamente às famílias atendidas pelos serviços. Foram padronizadas pelo grupo de trabalho as seguintes dietas: padrão, hiperproteica, para diabetes (dieta padrão e dieta completa), para gastrostomia, dieta à base de legumes e dieta com ingredientes da cesta básica, totalizando sete. 
Tabela 1. Ingredientes das dietas padronizadas para terapia de nutrição enteral. CampinasSP, 2010.

\begin{tabular}{|c|c|c|c|c|c|c|}
\hline Padrão 1 & Padrão 2 & Diabetes 1 & Diabetes 2 & Legumes & Gastrostomia & Cesta básica \\
\hline $\begin{array}{c}\text { Extrato de } \\
\text { soja }\end{array}$ & $\begin{array}{l}\text { Extrato de } \\
\text { soja }\end{array}$ & $\begin{array}{l}\text { Extrato de } \\
\text { soja }\end{array}$ & $\begin{array}{l}\text { Extrato de } \\
\text { soja }\end{array}$ & $\begin{array}{l}\text { Batata } \\
\text { cozida }\end{array}$ & $\begin{array}{c}\text { Leite } \\
\text { desnatado } \\
\text { fluido }\end{array}$ & $\begin{array}{c}\text { Leite } \\
\text { Integral } \\
\text { fluido }\end{array}$ \\
\hline $\begin{array}{l}\text { Óleo de } \\
\text { soja }\end{array}$ & $\begin{array}{l}\text { Óleo de } \\
\text { soja }\end{array}$ & Óleo de soja & Óleo de soja & $\begin{array}{c}\text { Cenoura } \\
\text { cozida }\end{array}$ & $\begin{array}{l}\text { Açúcar } \\
\text { refinado }\end{array}$ & Óleo de soja \\
\hline $\begin{array}{l}\text { Açúcar } \\
\text { refinado }\end{array}$ & $\begin{array}{c}\text { Leite } \\
\text { desnatado } \\
\text { em pó }\end{array}$ & $\begin{array}{c}\text { Amido } \\
\text { de milho } \\
\text { gelatinizado }\end{array}$ & $\begin{array}{c}\text { Leite } \\
\text { desnatado } \\
\text { em pó }\end{array}$ & $\begin{array}{c}\text { Leite } \\
\text { integral } \\
\text { fluido }\end{array}$ & Fubá & $\begin{array}{c}\text { Clara de ovo } \\
\text { cozida }\end{array}$ \\
\hline $\begin{array}{l}\text { Albumina } \\
\text { em pó }\end{array}$ & $\begin{array}{l}\text { Albumina } \\
\text { em pó }\end{array}$ & $\begin{array}{l}\text { Albumina } \\
\text { em pó }\end{array}$ & $\begin{array}{l}\text { Albumina } \\
\text { em pó }\end{array}$ & $\begin{array}{c}\text { Óleo de } \\
\text { soja }\end{array}$ & Arroz cozido & $\begin{array}{l}\text { Açúcar } \\
\text { refinado }\end{array}$ \\
\hline Sal & $\begin{array}{l}\text { Açúcar } \\
\text { refinado }\end{array}$ & $\begin{array}{c}\text { Maçã sem } \\
\text { casca e } \\
\text { cozida }\end{array}$ & $\begin{array}{c}\text { Amido } \\
\text { de milho } \\
\text { gelatinizado }\end{array}$ & $\begin{array}{l}\text { Farinha } \\
\text { de trigo } \\
\text { torrada }\end{array}$ & Óleo de soja & Fubá \\
\hline \multirow{3}{*}{$\begin{array}{c}\text { Água } \\
\text { filtrada } \\
\text { para } \\
\text { liquidificar }\end{array}$} & $\begin{array}{c}\text { Maçã sem } \\
\text { casca e } \\
\text { cozida }\end{array}$ & Sal & $\begin{array}{c}\text { Maçã sem } \\
\text { casca e } \\
\text { cozida }\end{array}$ & $\begin{array}{l}\text { Açúcar } \\
\text { refinado }\end{array}$ & $\begin{array}{c}\text { Carne de boi } \\
\text { magra }\end{array}$ & Sal \\
\hline & Sal & $\begin{array}{c}\text { Água } \\
\text { filtrada para } \\
\text { liquidificar }\end{array}$ & Sal & $\begin{array}{c}\text { Albumina } \\
\text { em pó }\end{array}$ & Batata cozida & $\begin{array}{c}\text { Água } \\
\text { filtrada para } \\
\text { liquidificar }\end{array}$ \\
\hline & $\begin{array}{c}\text { Água } \\
\text { filtrada } \\
\text { para } \\
\text { liquidificar }\end{array}$ & & $\begin{array}{c}\text { Água } \\
\text { filtrada } \\
\text { para } \\
\text { liquidificar }\end{array}$ & Sal & Cenoura cozida & \\
\hline
\end{tabular}


Na análise bromatológica, todas as formulações apresentaram-se homogêneas, com aspecto uniforme e sem separação de fases após três horas do preparo. Em relação à fluidez, as formulações passaram pelo equipo sem entupimento, sendo que a concentração de sólidos existentes em cada formulação permite o escoamento adequado da solução.

A tabela 2 apresenta os resultados dos cálculos nutricionais a partir das tabelas de composição de alimentos citadas. A tabela 3 apresenta a composição centesimal a partir dos resultados da análise bromatológica.

Tabela 2. Composição das dietas - formulações para 1000Kcal a partir dos cálculos com as tabelas de composição de alimentos padronizadas. Campinas-SP, 2010.

\begin{tabular}{cccccccc}
\hline Composição & $\begin{array}{c}\text { Padrão } \\
\mathbf{1}\end{array}$ & $\begin{array}{c}\text { Padrão } \\
\mathbf{2}\end{array}$ & $\begin{array}{c}\text { Diabetes } \\
\mathbf{1}\end{array}$ & $\begin{array}{c}\text { Diabetes } \\
\mathbf{2}\end{array}$ & Legumes & Gastrostomia & $\begin{array}{c}\text { Cesta } \\
\text { básica }\end{array}$ \\
\hline $\begin{array}{c}\text { Energia } \\
\text { (kcal) }\end{array}$ & 1100,5 & 1062,55 & 1144,63 & 1002,82 & 928,86 & 1073,93 & 1005,55 \\
$\begin{array}{c}\text { Proteína } \\
\quad(g)\end{array}$ & 40,24 & 43,22 & 46,48 & 47,6 & 35,09 & 52,43 & 33,61 \\
$\begin{array}{c}\text { Lipídeo } \\
\text { (g) }\end{array}$ & 39,58 & 32,79 & 40,18 & 32,82 & 33,12 & 39,57 & 30,65 \\
$\begin{array}{c}\text { Carboidrato } \\
\text { (g) }\end{array}$ & 145,83 & 148,65 & 149,27 & 129,27 & 122,62 & 127,03 & 148,81 \\
\hline
\end{tabular}

Tabela 3. Composição das dietas - formulações para $1.000 \mathrm{kcal}$ a partir da análise bromatológica. Campinas-SP, 2010.

\begin{tabular}{cccccccc}
\hline Composição & $\begin{array}{c}\text { Padrão } \\
\mathbf{1}\end{array}$ & $\begin{array}{c}\text { Padrão } \\
\mathbf{2}\end{array}$ & $\begin{array}{c}\text { Diabetes } \\
\mathbf{1}\end{array}$ & $\begin{array}{c}\text { Diabetes } \\
\mathbf{2}\end{array}$ & Legumes & Gastrostomia & $\begin{array}{c}\text { Cesta } \\
\text { básica }\end{array}$ \\
\hline $\begin{array}{c}\text { Energia } \\
(\text { kcal })\end{array}$ & 847,73 & 818,54 & 834,62 & 612,30 & 768,40 & 509,62 & 781,69 \\
$\begin{array}{c}\text { Proteína } \\
(\mathrm{g})\end{array}$ & 33,95 & 46,40 & 35,50 & 37,51 & 31,48 & 23,00 & 30,99 \\
$\begin{array}{c}\text { Lipídeo } \\
(\mathrm{g})\end{array}$ & 34,19 & 28,73 & 33,64 & 22,63 & 25,90 & 22,14 & 27,41 \\
$\begin{array}{c}\text { Carboidrato } \\
(\mathrm{g})\end{array}$ & 126,51 & 128,37 & 124,09 & 92,77 & 125,93 & 71,83 & 125,98 \\
\hline
\end{tabular}


Para as dietas mais utilizadas (padrão 1, diabetes 1 e diabetes 2, padrão 2), as médias das diferenças entre o cálculo e análise bromatológica foram 16\%, 14,7\%, 29,8\% e 10,4\%, respectivamente, o que demonstra a importância do ajuste destas dietas para suprir as necessidades dos pacientes.

Para as dietas que apresentam mais alimentos do que módulos (dieta de legumes, dieta com ingredientes da cesta básica e dieta para gastrostomia), as médias das diferenças foram 11,6\%, $14 \%$ e $49 \%$. A dieta de gastrostomia, por conter mais alimentos e carne, apresenta maior perda no processo de coar a formulação, o que justifica a diferença importante dos resultados.

Escolher e adequar as fontes de nutrientes para dietas não industrializadas é tarefa difícil e complexa para os nutricionistas. Além de adequadas quanto à composição nutricional e quanto à qualidade microbiológica, é preciso definir e facilitar as orientações para o manipulador e para a família. As dietas padronizadas apresentam ingredientes de fácil aquisição, praticidade de preparo, menor custo e menor contaminação, garantindo a segurança alimentar pela nutrição enteral, podendo ser prescritas para os pacientes atendidos pelos serviços citados do SUS.

\section{Perspectivas}

Após a comparação dos resultados da análise de composição centesimal das dietas padronizadas pelos cálculos e pela análise bromatológica, estão sendo realizados os ajustes e alterações dessas dietas para que possam atingir de fato a necessidade nutricional destes pacientes e sejam viáveis do ponto de vista do preparo, custo e entendimento do cuidador e familiares atendidos pelos serviços do SUS.

Além disso, o grupo pretende realizar a divulgação e a publicação do protocolo padronizado para hospitais e serviços de saúde do município de Campinas-SP e região, além de encaminhar este protocolo ao Ministério da Saúde.

\section{Agradecimentos}

Agradecimentos pela colaboração e participação neste projeto às nutricionistas Edma Maria de Araújo, Luciane C.R.Giordano, Maristela Talamoni, Marli Oliveira e Rosana Castelli Candido. 


\section{Referências}

1. Brasil. Ministério da Saúde. Portaria MS/GM no 2527 de 27 de outubro de 2011. Redefine a atenção domiciliar no âmbito do Sistema Único de Saúde - SUS. Diário oficial da União 27 out. 2011; (1):44.

2. Araújo EM, Menezes HC. Formulações com alimentos convencionais para nutrição enteral ou oral. Ciênc. Tecnol. Aliment. 2006; 26(3):533-38.

3. Arvanitakis M, Beck A, Coppens P, De Man F, Elia M, Hebuterne X, et al. Nutrition in care homes and home care: How to implement adequate strategies (report of the Brussels Forum 22-23 November 2007). Clin. Nutr. 2008; 27(4):481-88.

4. Hitchings $\mathrm{H}$, Best $\mathrm{C}$, Steed I. Home enteral tube feeding in older people: consideration of the issues. Br. J. Nurs. 2010; 19(18):1150-54.

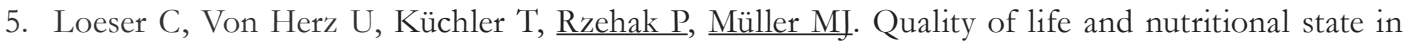
patients on home enteral tube feeding. Nutrition 2003; 19(7-8):605-11.

6. Waitzberg DL. Nutrição oral, enteral e parenteral na prática clínica. $3^{a}$ ed. São Paulo: Atheneu; 2002.

7. Zaban ALRS. Nutrição enteral domiciliar: um novo modelo de gestão econômica do Sistema Único de Saúde [Dissertação]. Brasília: Universidade de Brasília; 2009.

8. Brasil. Agência Nacional de Vigilância Sanitária. Resolução da Diretoria Colegiada no 63 , de 6 de julho de 2000. Regulamento técnico para fixar os requisitos mínimos exigidos para a terapia de nutrição enteral. Diário Oficial da União 2000.

9. Henrique GS, Rosado GP. Formulação com dietas enterais artesanais e determinação da osmolalidade pelo método crioscópico. Rev. Nutr. 1999; 12(3):225-32.

10. Menegassi B, Santana LS, Coelho JC, Martins AO, Pinto JPAN, Costa TMB, et al. Características físico-químicas e qualidade nutricional de dietas enterais não-industrializadas. Alim. Nutr. 2007; 18(2):127-32.

11. Mitne C. Preparações não-industrializadas para nutrição enteral. In: Waitzberg DL, organizador. Nutrição oral, enteral e parenteral na prática clínica. v. 1.3 ed. São Paulo: Atheneu; 2000. p. 629-40.

12. Baxter YC, Waitzberg DL. Fórmulas enterais: complexidades de nutrientes e categorização. In: Silva SMC, Mura JDP. Tratado de alimentação, nutrição e dietoterapia. São Paulo: Roca; 2007. p. 883-892.

13. Borges VC, Waitzberg DL, Silva M LT, Bottoni A, et al. Nutrição domiciliar: uma experiência no Brasil. In: Waitzberg DL, organizador. Nutrição oral, enteral e parenteral na prática clínica. v. 2. 3. ed. São Paulo: Atheneu; 2004. p. 977-987.

14. Universidade Estadual de Campinas. Núcleo de Estudos e Pesquisas em Alimentação. Tabela brasileira de composição de alimentos. Versão II. 2. ed. Campinas, SP: NEPA-UNICAMP; 2006. 113 p.

15. U.S. Department of Agriculture, Agricultural Research Service. USDA National Nutrient Database for Standard Reference [Internet]. Disponível em: http://www.ars.usda.gov/Services/docs. htm?docid $=8964$ 
16. Mendez MHM, Derivi SCN, Rodrigues MCR, Fernandes ML. Tabela de composição de alimentos. Niterói: Universidade Federal Fluminense; 1995.

17. Pearson D. Tecnicas de laboratórios para el análisis de alimentos. Zaragoza: Acribia; 1976. 331 p.

18. Horwitz W. Official methods of analysis. $11 \mathrm{ed}$. Washington DC: Association of Official Analytical Chemists; 1975.

19. Cunniff PA. Official methods of analysis. 16. ed. Washington DC: Association of Official Analytical Chemists; 1995.

20. Bligh EG, Dyer WJ. A rapid method of total lipid extraction and purification. Can. J. Biochem. Physiol. 1959; 37(8):911-917.

21. Lees R. Manual de análises de alimentos. Zaragoza: Acribia; 1979.

Recebido: 16/4/2014

Revisado: 29/5/2014

Aprovado: 16/6/2014 